\title{
A Low Overhead Pilot-Aided Parameter Estimation Scheme for Asymmetric PCMA Systems
}

\author{
Fei He, Xiang Chen, Jian Yan, Ming Zhao, Shidong Zhou \\ State Key Laboratory on Microwave and Digital Communications \\ Tsinghua National Laboratory for Information Science and Technology \\ Department of Electronic Engineering, Tsinghua University, Beijing 100084, China \\ Email: \{flyingpossible, chenxiang98\}@ gmail.com
}

\begin{abstract}
A pilot-aided scheme for the parameter estimation in Paired Carrier Multiple Access (PCMA) satellite communication systems is proposed in this paper. Recently, the asymmetric application mode of PCMA, which is more efficient and pragmatic than the symmetric mode, has been studied with great interest. In our research, carefully selected pilot sequences have been inserted into the frame in an attempt to achieve better estimation performance with only a very low overhead. The channel estimation method, which is based on Wiener optimal filtering, is also derived with respect to the (quasi-)static selective fading channel environment. Different from the foregoing work, the key result of this paper is the construction of a single module which jointly implements various parameter estimation modules as a whole. As shown in the simulation results, the proposed scheme outperforms the conventional data-aided scheme, especially in the high Signal to Noise Ratio (SNR) scenario which is typical in asymmetric PCMA systems.
\end{abstract}

\section{INTRODUCTION}

Paired Carrier Multiple Access (PCMA) is a new multiple access technique in two-way satellite communications, which was first proposed by ViaSat Inc. in 1998 [1]. PCMA allows two different earth stations to use the same frequency, time slots and Code Division Multiple Access (CDMA) codes at the same time. It can effectively double the capacity of satellite systems with the same resource. For PCMA, two assumptions are required for satellite links [1]: first, the satellite operates in a loopback mode, which means the signal transmitted by each terminal can also be received by itself; second, the satellite works as a bent-pipe transponder, with simply power amplification and frequency translation. These requirements are valid in most cases.

A PCMA system allocates the same spectrum band to a pair of satellite terminals. However, taking advantage of the loopback and bent-pipe nature, each terminal can reliably estimate and separate its own component in the composite downlink, since each terminal knows exactly what information it sent. Under perfect estimation assumption, the desired signal in the composite downlink can be obtained when its own sent part is subtracted with little remnant self-interference.

There are two basic modes in the application of PCMA, the symmetric mode and the asymmetric mode. In the symmetric

This work is partially supported by National Basic Research Program of China (2007CB31060), China's Major Project (2009ZX03003-009), NCET05-0071, PCSIRT and China's 863 Project (2009AA011501).

CHINACOM 2010, August 25-27, Beijing, China

Copyright $\odot 2011$ ICST 973-963-9799-97-4

DOI 10.4108/chinacom.2010.34 mode, the transmission powers are almost equal for a pair of terminals in the PCMA system; thus the self-interference cancellation should be performed at both sides in order to get desired signals. In the asymmetric mode, the base station transmits much higher power than remote terminals. The common demodulation technique for desired signals can be easily carried out at remote terminals. But the power of received desired signals at the base station is far under that of self-interference, and the self-interference cancellation is indispensable.

The loopback parameters, including round trip propagation delay, amplitude, carrier frequency offset (CFO), carrier phase offset, symbol timing offset, and signal processing filter response, must be perfectly estimated before reconstructing and subtracting the self-interference. As summarized in [2], these various estimation tasks have been studied in a moduleby-module fashion. Timing synchronization module as the primary condition of other estimations has been widely discussed. However, these estimation methods only utilize the characteristic of autocorrelation of local known data, and the PCMA system application mode is set in the symmetric mode only. The common data-aided scheme has poor performance and its convergence is slow. Some further study on parameter estimation modules for amplitude, carrier phase offset, and CFO can be find respectively in [3] [4] [5]. Pan studied the performance degradation caused by timing error in [6].

Since PCMA system needs estimation work at both side in the symmetric mode, the asymmetric mode may be more economical and pragmatic. The non-cooperative signal interception in the asymmetric scenario has been studied in [7]. In fact, ViaSat Inc. has also proposed some practical implementation methods based on training sequences before real twoway communication in an attempt to get good parameter estimation. Following this idea, we propose a pilot-aided estimation method for asymmetric PCMA systems in this paper, which can enhance the estimation performance remarkably with very low pilot overhead.

Channel estimation based on complementary sequences for (quasi-)static intersymbol interference (ISI) channel has been tackled in [8]. A "two-sided" channel estimation method is introduced to minimize the normalized maximum-likelihood (ML) estimation variance, in which the paired complementary 
sequences called a preamble and a postamble are put at the head and rear of each estimation block respectively. More discussions on data-aided channel estimation for single carrier selective fading channel can be found in [9]. They focus on the accurate bound for the variance of ML estimation method, and deal with the joint part of pilot and data symbols with too much care. A practical method based on Wiener filtering with acceptable complexity will be introduced in this paper.

In the following discussion of this paper, only the asymmetric mode is considered and only the estimation at the base station is cared about. In Section II, the asymmetric PCMA system and its channel model are described in detail, including the frame structure and the consideration for pilot selection. Section III addresses the channel estimation problem in our system based on Wiener optimal filtering. Section IV presents the simulation results, which indicate improvements with the pilot-aided scheme over the data-aided one. The paper is concluded in Section V.

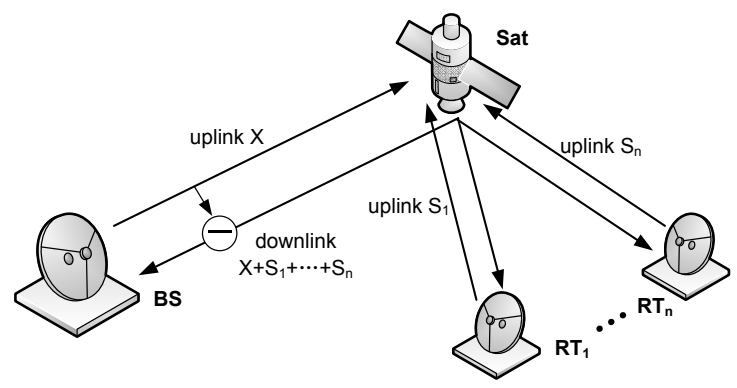

Fig. 1. Network structure in asymmetric PCMA system

\section{SYSTEM DESCRIPTION}

Fig. 1 shows a typical asymmetric scenario in the PCMA satellite communication system, which contains a Base Station (BS) and $n$ distributed Remote Terminals (RTs). The uplink of BS is the big carrier signal $X$ with wider bandwidth and higher power than $S_{n}$, the uplink of $\mathrm{RT}_{n}$. The frequency reuse status is illustrated in Fig. 2. All the receivers at BS and RTs can receive the composite downlink signal $X+S_{1}+\cdots+S_{n}$, which is transported by the bent-pipe Satellite (Sat). For RTs, the desired signal is the big carrier $X$, and the high power level guarantees the common demodulation without self-interference cancellation. For BS, the situation is quite different. In order to correctly demodulate the desired signal with small power and narrow bandwidth, we first subtract the big carrier $X$ from the composite downlink, and bandpass filter the processed downlink into the corresponding band allocated for the given RT user in advance. Because of the limit of power and terrain, any direct communication between RT users without BS and Sat is impossible. The information exchanging between different RT users should be carried out at BS. We redefine the link RT $\rightarrow$ Sat $\rightarrow \mathrm{BS}$ as $u p$-link, and $\mathrm{BS} \rightarrow \mathrm{Sat} \rightarrow \mathrm{RT}$ as down-link. The bent-pipe nature of satellite simplifies the description of communication process using the concepts of up-link and down-link without considering Sat any more.

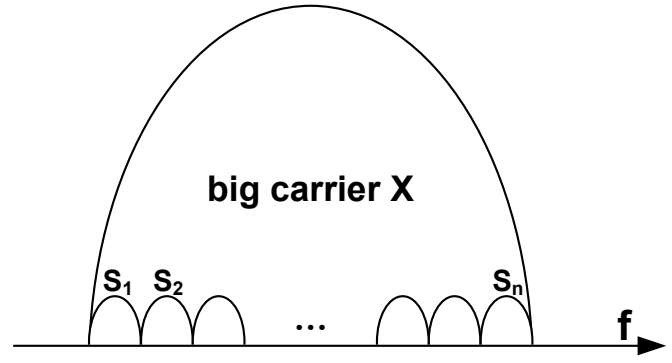

Fig. 2. Frequency reuse in asymmetric PCMA system

\section{A. Channel Model}

Although the satellite link can be modeled as an AWGN channel, the frequency response of signal processing filters, such as shaping filters at transmitters and receivers, brings the frequency selectivity to this system. After obtaining the accurate timing synchronization and CFO, we take all the other parameters as a general joint channel response at the base station. A single carrier selective fading channel environment is to be dealt with, and it is time-invariant ignoring the effect of phase noise.

Consider the complex baseband-equivalent representation of linear time-invariant channel with $M$ distinct paths and impulse response

$$
h(\tau)=\sum_{i=0}^{M-1} \alpha_{i} \delta\left(\tau-\tau_{i}\right),
$$

where the $\tau_{i}$ s are the delays of the virtual individual paths caused by frequency selectivity in our system, which, different from the model order $M$, could not be known or estimated. However, we can assume the path gains $\alpha_{i}$ are zero mean stochastic processes with normalized overall power, i.e.,

$$
E\left\{\alpha_{i}\right\}=0, \quad \sum_{i=0}^{M-1} E\left\{\left|\alpha_{i}\right|^{2}\right\}=\sum_{i=0}^{M-1} \sigma_{i}^{2}=1
$$

and wide-sense stationary uncorrelated scattering (WSSUS) [10], i.e., the cross-covariance $\varphi_{i_{1} i_{2}}\left(t_{1}, t_{2}\right)$ of $\alpha_{i_{1}}$ and $\alpha_{i_{2}}$ at time instances $t_{1}$ and $t_{2}$ is

$$
\begin{aligned}
\varphi_{i_{1} i_{2}}\left(t_{1}, t_{2}\right) & =E\left\{\alpha_{i_{1}}\left(t_{1}\right) \alpha_{i_{2}}^{*}\left(t_{2}\right)\right\} \\
& =\varphi_{i_{1}}\left(t_{1}-t_{2}\right) \delta\left(i_{1}-i_{2}\right),
\end{aligned}
$$

where $\varphi_{i_{1}}\left(t_{1}, t_{2}\right)=\varphi_{i_{1}}(\tau)=\sigma_{i_{1}}^{2}$ follows the wide-sense stationary of $\alpha_{i}(t)=\alpha_{i}$. The Fourier transform of the autocovariance $\varphi_{i}(\tau)$ is the ideal impulse power spectrum $\Phi_{i}(f)$ without any Doppler shift.

\section{B. Frame Design}

As emphasized above, we only care about estimation of big carrier $X$, and the frame structure of down-link should be focused on. Fig. 3 illustrates the down-link frame structure, in which, $P_{1}$ is a single tone sequence of length $N_{1}, P_{2}$ of length $2 N_{2}$ is concatenated by a pair of Golay complementary 


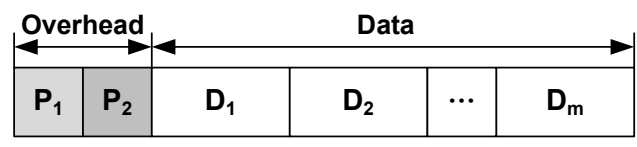

Fig. 3. Frame structure of down-link

sequences [11]. The rest of this frame is divided into $m$ time slots, each of which can be used by a pair of RT users at the same time by taking the advantage of network coding in physical layer. At the base station, the network coding can be simply implemented by XOR operation in bit level after demodulation and decoding; the RT users can redo the XOR operation with local known bits to get the new information. This technique doubles the capacity of our system.

Assume we can support $m$ communication pairs of users at most, and the ratio of overhead is

$$
R_{\text {overhead }}=\frac{N_{1}+2 N_{2}}{N_{1}+2 N_{2}+m N_{d a t a}},
$$

where $N_{\text {data }}$ is the number of symbols in each time slot $D_{i}$. In our practical system, a list of parameters is $N_{1}=2048, N_{2}=$ $1024, N_{\text {data }}=25000, m=10$; so $R_{\text {overhead }}=1.61 \%$.

The single tone sequence $P_{1}$ is useful for coarse timing capture with consecutive FFT computations on received samples. Meanwhile, the coarse CFO can be estimated by calculating the position shift of the given single tone. Considering the properties of pilot $P_{2}$, a fast Golay correlator is used to complete the fine timing synchronization and more accurate estimation of CFO, as well as the general channel response.

\section{Pilot Selection}

The selection of pilot $P_{2}$ cares about the following issues: the sidelobes of auto-correlation spectrum of the pilot sequence should be as low as possible, which is important for accurate synchronization in low SNR scenario and the channel estimation; the correlator or matched filter can be implemented efficiently for the chosen pilot sequence, and that is the key to meet the real time requirement in the high data rate scenario.

Golay complementary sequences satisfy these good characteristics [11]. Let $\boldsymbol{a}=\left(a_{0}, a_{1}, \ldots, a_{N-1}\right)$ be a bipolar sequence of length $N$ such that $a_{i} \in\{+1,-1\}$. Define the Aperiodic Auto-Correlation Function (AACF) of $\boldsymbol{a}$ by

$$
\rho_{\boldsymbol{a}}(k)=\sum_{i=0}^{N-k-1} a_{i} a_{i+k}, \quad 0 \leq k \leq N-1 .
$$

Let $\boldsymbol{b}$ be defined similarly to $\boldsymbol{a}$. The pair $(\boldsymbol{a}, \boldsymbol{b})$ is called a Golay Complementary Pair (GCP) if:

$$
\rho_{\boldsymbol{a}}(k)+\rho_{\boldsymbol{b}}(k)=0, \quad k \neq 0 .
$$

Each member of a GCP is called a Golay Complementary Sequence (GCS).

There is already an efficient method to perform correlation of an data stream with a GCS of length $N$ achieving a complexity of $2 \log _{2}(N)$ operations per sample, in comparison with $N$ operations per sample for direct correlation. Although the property indicated by (6) has not been made full use of, the single GCS has been adopted as the Primary Synchronization Code (PSC) in WCDMA systems to acquire the slot synchronization.

In our system, we take a GCP of length $N_{2}$ to concatenate the pilot $P_{2}$. With an off-the-shelf complex PSC $\boldsymbol{a}$ in WCDMA systems and its fast Golay correlator, we construct the corresponding member $\boldsymbol{b}$ of the same GCP easily, and reuse the correlator for the new concatenated sequence of length $2 N_{2}$ by making full use of the discarded interim correlation results without additional computation cost.

\section{Channel Estimation}

Using the frame structure proposed for down-link in Section II-B, we can acquire the accurate timing synchronization with little remnant $\mathrm{CFO}$ not more than the spectrum resolution of FFT computation for the pilot $P_{1}$. Since the pilot and data symbol sequences are all known at BS, the estimation of remnant CFO can be solved by classical estimators such as Kay's estimator for a single frequency [12]. The general joint channel response could be addressed until the estimated CFO has been compensated.

When the channel can be described by a WSSUS model as in Section II-A, using FIR Wiener filters is a good choice to reduce the rank of estimators. Two cascaded one-dimensional and two-dimensional Wiener filtering have been investigated in [13] and [14] for multi-carrier modulation systems, and the rapid time-variant case has also been addressed in [10]. In fact, the application of Wiener filtering theory on the single carrier selective fading channel has been discussed in [15]. All the above methods assume the known power spectrum and other key statistical parameters such as maximum Doppler frequency and maximum echo delay, and the SNR also should be estimated in advance. Unfortunately, those are not available in our case. The frequency selectivity in our asymmetric PCMA system mainly comes from the frequency response of artificial filters with time-invariant nature ignoring the effect of phase noise. The uniform delay power spectrum can be taken as the worst case, but suitable maximum echo delay is difficult to decide.

In this paper, we do not rely on the numerical assumption of channel parameters from statistic of measurements, but make full use of the correlation characteristic of sequences in time domain. First, a general data-aided scheme is derived. Then, the pilot-aided case is discussed. The performance of two schemes will be compared by simulations in Section IV later.

\section{A. Wiener Optimal Filtering}

Consider the original statistical filtering problem described in [16]. The filter input is denoted by the time series $\boldsymbol{u}=$ $[u(0), u(1), u(2), \ldots]^{T}$, and the impulse response of the filter is denoted by $\boldsymbol{w}=\left[w_{0}, w_{1}, w_{2}, \ldots\right]^{T}$, both of which are assumed to have complex values and infinite duration. The filter output $y(n)$ at discrete time $n$ is defined by the linear 
convolution sum:

$$
y(n)=\sum_{k=0}^{\infty} w_{k}^{*} u(n-k), \quad n=0,1,2, \ldots,
$$

where the $[\cdot]^{*}$ denotes complex conjugation.

The purpose of the Wiener filter is to produce an estimation of the desired response, $d(n)$. With the assumption of WSSUS channel model, moreover, we can assume the filter input and the desired response are single realizations of jointly widesense stationary stochastic processes, both with zero mean. To optimize the filter design, we choose to minimize the meansquare values of the estimation error $e(n)=d(n)-y(n)$, and define the cost function as the mean-squared error (MSE):

$$
J(\boldsymbol{w})=E\left\{e(n) e^{*}(n)\right\}=E\left\{|e(n)|^{2}\right\} .
$$

\section{B. Wiener-Hopf Equations}

The principle of orthogonality specifies the necessary and sufficient condition for the optimum operation of the filter with respect to the cost function $J(\boldsymbol{w})$, that is

$$
E\left\{u(n-k) e_{o}^{*}(n)\right\}=0, \quad k=0,1,2, \ldots,
$$

where $e_{o}$ denotes the special value of the estimation error that results when the filter operates in its optimum condition. We can rewrite (9) by substituting (7), expanding and rearranging terms as

$$
\begin{array}{r}
\sum_{i=0}^{\infty} w_{o i} E\left\{u(n-k) u^{*}(n-i)\right\}=E\left\{u(n-k) d^{*}(n)\right\}, \\
k=0,1,2, \ldots,
\end{array}
$$

where $w_{o i}$ is the $i$ th coefficient in the impulse response of the optimum filter. (10) are called the Wiener-Hopf equations.

The solution of the set of Wiener-Hopf equations is greatly simplified in the special case when the FIR filter is used to perform the estimation of desired response. The impulse response of the FIR filter is defined by the finite set of tap weights $\boldsymbol{w}=\left[w_{0}, w_{1}, \ldots, w_{L-1}\right]^{T}$. Let $\boldsymbol{R}$ denote the $L \times L$ correlation matrix of the tap inputs:

$$
\begin{aligned}
\boldsymbol{R} & =E\left\{\boldsymbol{u}(n) \boldsymbol{u}^{H}(n)\right\} \\
& =\left[\begin{array}{cccc}
r(0) & r(1) & \cdots & r(L-1) \\
r^{*}(1) & r(0) & \cdots & r(L-2) \\
\vdots & \vdots & \ddots & \vdots \\
r^{*}(L-1) & r^{*}(L-2) & \cdots & r(0)
\end{array}\right],
\end{aligned}
$$

where $\boldsymbol{u}(n)$ is the $L \times 1$ tap-input vector,

$$
\boldsymbol{u}(n)=[u(n), u(n-1), \ldots, u(n-L+1)]^{T},
$$

and $r(k)$ is the autocorrelation function of the filter input for a lag of $k$,

$$
r(k)=E\left\{u(n) u^{*}(n-k)\right\}, \quad k=0,1, \ldots, L-1 .
$$

Correspondingly, let $\boldsymbol{p}$ denotes the $L \times 1$ cross-correlation vector between the tap inputs of the filter and the desired response $d(n)$ :

$$
\begin{aligned}
\boldsymbol{p} & =E\left\{\boldsymbol{u}(n) d^{*}(n)\right\} \\
& =[p(0), p(-1), \ldots, p(1-L)]^{T},
\end{aligned}
$$

where $p(-k)=E\left\{u(n-k) d^{*}(n)\right\}$. We may thus rewrite the Wiener-Hopf equations (10) in a compact matrix form:

$$
R w_{o}=p,
$$

where $\boldsymbol{w}_{\boldsymbol{o}}=\left[w_{o, 0}, w_{o, 1}, \ldots, w_{o, L-1}\right]^{T}$ denotes the $L \times 1$ optimum tap-weight vector of the FIR filter.

\section{Data-Aided Scheme}

In our channel estimation problem, the original unshaped down-link symbols are corresponding to the inputs $u(n)$; the received composite downlink samples are corresponding to the desired response $d(n)$; the general joint channel response $\boldsymbol{h}$ to be estimated is $\boldsymbol{w}_{\boldsymbol{o}}$, the solution of (15).

As $\boldsymbol{R}$ is a Hermitian Toeplitz matrix, only $L$ autocorrelation values $r(k)$ of (13) need to be calculated, where $L$ is the finite length of $\boldsymbol{w}_{\boldsymbol{o}}$. The expectation operation can be substituted by the summation on known $u(n)$ like (5), with an additional complex conjugation operation on the latter item, i.e.,

$$
\hat{r}(k)=\sum_{n=k}^{N-1} u(n) u^{*}(n-k), \quad k=0,1, \ldots, L-1,
$$

where $N$ is the total number of known symbols. The similar calculation is done for each term of $\boldsymbol{p}$ in (14). Considering the special structure of Hermitian Toeplitz matrix, (15) can be solved efficiently with Hermitian Levinson recursive algorithm [17]. For simplification, only the real part of $\boldsymbol{R}$ is considered, which then is a symmetric Toeplitz matrix, and the more efficient Classic Levinson recursive algorithm can be adopted.

\section{Pilot-Aided Scheme}

After we calculate values of AACF by (16) for the GCP in pilot sequence $P_{2}$ defined in Section II-C, two sets of linear equations for the general joint channel response $\boldsymbol{h}$ are established, that are

$$
\begin{aligned}
\boldsymbol{R}_{\boldsymbol{a}} \boldsymbol{h} & =\boldsymbol{p}_{\boldsymbol{a}}, \\
\boldsymbol{R}_{\boldsymbol{b}} \boldsymbol{h} & =\boldsymbol{p}_{\boldsymbol{b}} .
\end{aligned}
$$

Considering the property indicated by (6), the solution $\boldsymbol{h}$ is trivial:

$$
\begin{aligned}
\boldsymbol{h} & =\boldsymbol{I}_{L} \boldsymbol{h} \\
& =\left(\boldsymbol{R}_{\boldsymbol{a}}+\boldsymbol{R}_{\boldsymbol{b}}\right) \boldsymbol{h} \\
& =\boldsymbol{p}_{\boldsymbol{a}}+\boldsymbol{p}_{\boldsymbol{a}},
\end{aligned}
$$

where $\boldsymbol{I}_{L}$ is an $L \times L$ identity matrix.

\section{Simulation Results}

We recall the down-link frame structure and the parameters listed in Section II-B. The pilot $P_{2}$ and all the data symbols are in QPSK constellation, while the pilot $P_{1}$ are special complexvalued single tone signals constructed from the inverse FFT manipulation. We focus on the performance of channel estimation. Since our estimation method does not require any statistic of the channel model or preestimated SNR, what we only need to set is a big enough $L$. In the following simulations, this setting is $L=101$. 


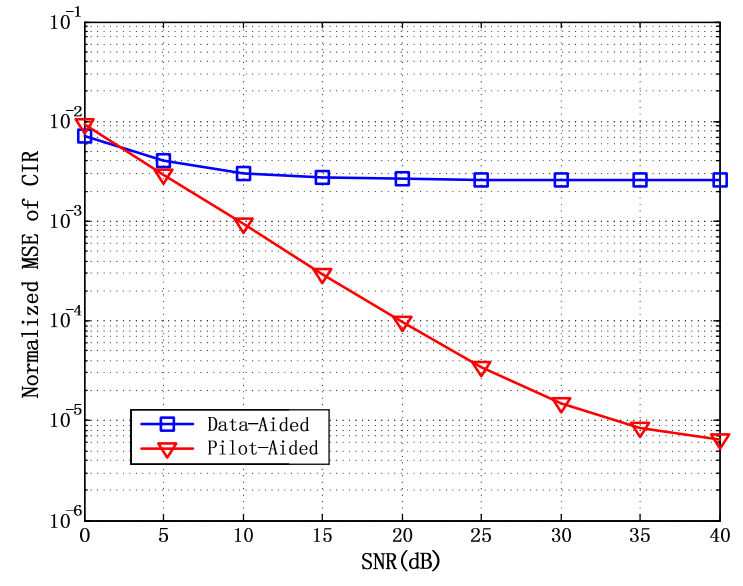

Fig. 4. Normalized MSE of channel impulse response

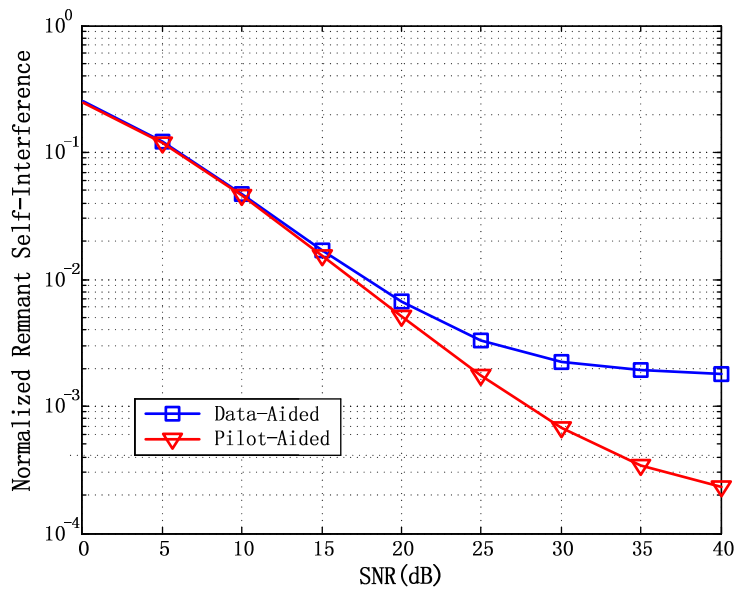

Fig. 5. Normalized remnant self-interference

Fig. 4 compares the normalized MSE of general joint channel impulse response $\boldsymbol{h}$ in the data-aided scheme and in the pilot-aided scheme; Fig. 5 shows the difference between the normalized remnant self-interference in two schemes.

Obviously, the pilot-aided scheme outperforms the dataaided one, especially in high SNR regime, which is typical at the base station in asymmetric PCMA systems. Considering the phase noise, however, the assumption that the general joint channel response is (quasi-)static in one down-link frame duration has been questioned. With uptodate estimated parameters, we reconstruct shaped samples from known down-link data symbols, and compare them with the received composite downlink in a relative short interval to estimate the phase noise, which must be compensated before demodulation and decoding. The simulation results also show that the estimation performances of $\mathrm{CFO}$ and channel response are coupled to some extent, so some iterative techniques should be considered to improve the overall performances of self-interference cancellation.

\section{CONCLUSION}

In this paper, we propose a special frame structure for the down-link of the asymmetric PCMA satellite communication systems. With the selected pilot, we have achieved improvements in the performance of parameter estimation in contrast with the conventional data-aided scheme: timing synchronization is quicker and more robust with large CFO considering the pilot $P_{1}$; the general joint channel impulse response can be estimated more accurately taking advantage of the superior autocorrelation characteristic of GCP which constitutes $P_{2}$. And it is only at the cost of very low overhead, which is less than $2 \%$ in our system. For the physical implementation of PCMA technique in satellite communications, this paper provides some useful references.

\section{REFERENCES}

[1] Mark Dankberg, "Paired Carrier Multiple Access (PCMA) for satellite communications," Pacific Telecommunications Conference, Honolulu Hawaii, January 13, 1998.

[2] Po Zhou, Jian Yan and Fengcai Zhou, "Introduction and application analysis for Paired Carrier Multiple Access techniuqe," Digital Communication World, no. 7, 2008, pp. 70-74. (in Chinese)

[3] Shenfu Pan et al., "Estimation of interference amplitude in PCMA system," Vacuum Electronics, no. 2, 2003, pp. 21-24. (in Chinese)

[4] Shenfu Pan et al., "Interference carrier phase estimation in PCMA system," Electronic Warfare, no. 6, 2004, pp. 8-11. (in Chinese)

[5] Liqiang Shao and Yang Zhang, "Estimation of carrier frequency offset in PCMA system," Science \& Technology Information, no. 14, 2006, pp. 13-14. (in Chinese)

[6] Shenfu Pan et al., "Performance degradation caused by timing error in PCMA system," Information Technology, vol. 27, no. 5, May. 2003, pp. 63-65. (in Chinese)

[7] Di Fu and Yong Gao, "Signal interception method for asymmetric PCMA satellite communication systems," Modern Electronics Technique, vol. 30, no. 7, Feb. 2007, pp. 28-30, 34. (in Chinese)

[8] P. Spasojevic and C. N. Georghiades, "Complementary sequences for ISI channel estimation," IEEE Trans. Inform. Theory, vol. 47, no. 3, March 2001, pp. 1145-1152.

[9] H. Meyr, M. Moeneclaey, and S. A. Fletchel, Digital Communication Receivers: Synchronization, Channel Estimation and Signal Processing, John Wiley \& Sons, 1997.

[10] C. Sgraja and J. Lindner, "Estimation of rapid time-variant channels for OFDM using Wiener filtering," Proc. IEEE Intl. Conf. Commun., vol. 4, Anchorage, AK, May 2003, pp. 2390-2395.

[11] M.G. Parker, K.G. Paterson and C. Tellambura, "Golay complementary sequences," in Wiley Encyclopedia ofTelecommunications, John G. Proakis, ed., Wiley, 2003.

[12] S. Kay, "A fast and accurate single frequency estimator," IEEE Trans. Acoustic, Speech, and Signal Proc., vol. 37, no. 12, Dec. 1989.

[13] P. Höher, "TCM on frequency-selective land-mobile fading channels," in Proc. Tirrenia Int. Workshop Digital Communications, Tirrenia, Italy, Sept. 1991, pp. 317-328.

[14] P. Höher, S. Kaiser, and P. Robertson, "Two-dimensional pilot-symbolaided channel estimation by Wiener filtering," in Proc. ICASSP-97, vol. 3, 1997, pp. 1845-1848.

[15] S. A. Fechtel and H. Meyr, "Optimal parametric feedforward estimation of frequency-selective fading radio channels," IEEE Trans. Commun., vol. 42, no. 2/3/4, Feb./Mar./Apr. 1994, pp. 1639-1650.

[16] S. Haykin, Adaptive Filter Theory, 4th ed. Prentice-Hall, 2002.

[17] H. Krishna and S. Morgera, "The Levinson recurrence and fast algorithms for solving Toeplitz systems of linear equations," IEEE Trans. ASSP, vol. 35, no. 6, Jun. 1987, pp. 839-848. 